\title{
On the Mazur-Ulam problem in fuzzy anti-normed spaces
}

\author{
Majid Abrishami-Moghaddam \\ Department of Mathematics, Birjand Branch, Islamic Azad University, Birjand, Iran \\ E-mail: m.abrishami.m@gmail.com
}

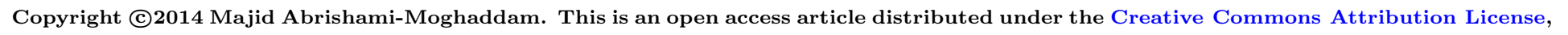
which permits unrestricted use, distribution, and reproduction in any medium, provided the original work is properly cited.

\begin{abstract}
The aim of this article is to proved a Mazur-Ulam type theorem in the strictly convex fuzzy anti-normed spaces.
\end{abstract}

Keywords: Fuzzy anti-normed space, Mazur-Ulam theorem, strictly convex.

\section{Introduction and preliminaries}

The theory of fuzzy sets was introduced by L. Zadeh [11] in 1965 and thereafter several authors applied it different branches of pure and applied mathematics. Many mathematicians considered the fuzzy normed spaces in several angels (see [1], [4], [10]). In [6] Iqbal H. Jebril and Samanta introduced fuzzy anti-norm on a linear space depending on the idea of fuzzy anti-norm was introduced by Bag and Samanta [2] and investigated their important properties. In 1932, the theory of isometric mappings was originated in the classical paper [8] by Mazur and Ulam. They proved that every isometry $\mathrm{f}$ of a normed real vector space $\mathrm{X}$ onto another normed real vector space $X$ is a linear mapping up to translation, that is, $x \mapsto f(x)-f(0)$ is linear, which amounts to the definition that $f$ is affine. We call this the Mazur-Ulam theorem. The property is not true for normed complex vector spaces. The hypothesis of surjectivity is essential. Without this assumption, Baker [3] proved that every isometry from a normed real space into a strictly convex normed real space is linear up to translation. A number of mathematicians have had deal with the Mazur-Ulam theorem; see [7, 9] and references therein. In this paper, we prove that the Mazur-Ulam theorem holds under some conditions in the fuzzy anti-normed spaces. We establish a Mazur-Ulam type theorem in the framework of strictly convex normed spaces by using some ideas of [5]. Now we recall some notations and definitions used in this paper.

Definition 1.1 [6] Let $X$ be a linear space over a real field $F$. A fuzzy subset $N$ of $X \times \mathcal{R}$ is called a fuzzy anti-norm on $X$ if the following conditions are satisfied for all $x, y \in X$

$\left(a-N_{1}\right)$ For all $t \in \mathcal{R}$ with $t \leq 0, N(x, t)=1$,

$\left(a-N_{2}\right)$ For all $t \in \mathcal{R}$ with $t>0, N(x, t)=0$ if and only if $x=\overline{0}$,

$\left(a-N_{3}\right)$ For all $t \in \mathcal{R}$ with $t>0, N(\alpha x, t)=N(x, t /|\alpha|)$, for all $\alpha \neq 0, \alpha \in F$,

$\left(a-N_{4}\right)$ For all $s, t \in \mathcal{R}, N(x+y, t+s) \leq \max \{N(x, s), N(y, t)\}$,

$\left(a-N_{5}\right) N(x, t)$ is a non-increasing function of $t \in \mathcal{R}$ and $\lim _{t \rightarrow \infty} N(x, t)=0$.

Then the pair $(X, N)$ is called a fuzzy anti-normed linear space. 
Example 1.2 Let $(X,\|\|$.$) be a normed space. If for all k, m, n \in \mathcal{R}^{+}$we define

$\mathcal{N}(x, t)=\left\{\begin{array}{cll}\frac{m\|x\|}{k t^{n}+m\|x\|} & \text { if } \quad t>0 \\ 1 & \text { if } \quad t \leq 0\end{array}\right.$

In particular if $k=m=n=1$ we have

$\mathcal{N}(x, t)=\left\{\begin{array}{cll}\frac{\|x\|}{t+\|x\|} & \text { if } \quad t>0 \\ 1 & \text { if } \quad t \leq 0\end{array}\right.$

which is called the standard fuzzy anti-norm induced by the norm $\|$.$\| .$

Definition 1.3 A fuzzy anti-normed space $X$ is called strictly convex if $N(x+y, s+t)=\max \{N(x, s), N(y, t)\}$ and $N(x, s)=N(y, t)$ implies that $x=y$ and $s=t$.

Definition 1.4 Let $(X, N)$ and $(Y, N)$ be two fuzzy anti-normed spaces. We call that $f:(X, N) \rightarrow(Y, N)$ is a fuzzy isometry if $N(x-y, s)=N(f(x)-f(y), s)$ for all $x, y \in X$ and $s>0$.

Definition 1.5 Let $X$ be a real linear space and $x, y, z$ mutually disjoint elements of $X$. Then $x, y$ and $z$ are said to be collinear if $y-z=k(x-z)$ for some real number $k$.

\section{Main results}

In this section we will prove that the MazurUlam theorem under some conditions in the fuzzy real anti-normed strictly convex spaces. First, we prove the following lemma that is require for the main theorem of our paper.

Lemma 2.1 Let $X$ be a fuzzy anti-normed space which is strictly convex and let $y, z \in X$ and $s>0$. Then $x=\frac{y+z}{2}$ is unique element of $X$ such that

$N(y-x, s)=N(y-z, 2 s)$

and

$N(z-x, s)=N(y-z, 2 s)$.

Proof. There is nothing to prove if $y=z$. Let $y \neq z$. Then by $\left(a-N_{3}\right)$, we have

$N(y-x, s)=N\left(y-\frac{y+z}{2}, s\right)=N(y-z, 2 s)$

and

$N(z-x, s)=N\left(z-\frac{y+z}{2}, s\right)=N(y-z, 2 s)$,

that is the existence holds. For the uniqueness, we may assume that $u$ and $v$ are two elements of $X$ such that $N(y-u, s)=N(y-v, s)=N(z-u, s)=N(z-v, s)=N(y-z, 2 s)$.

Then

$$
\begin{gathered}
N\left(y-\frac{u+v}{2}, s\right) \leq \max \{N(y-u, s), N(y-v, s)\} \\
=N(y-z, 2 s)
\end{gathered}
$$

and

$$
\begin{gathered}
N\left(z-\frac{u+v}{2}, s\right) \leq \max \{N(z-u, s), N(z-v, s)\} \\
=N(y-z, 2 s) .
\end{gathered}
$$


If both of inequalities (2.1) and (2.2) were strict we would have

$$
\begin{aligned}
N(y-z, 2 s) & =N\left(y-\frac{u+v}{2}+\frac{u+v}{2}-z, 2 s\right) \\
& \leq \max \left\{N\left(y-\frac{u+v}{2}\right), N\left(z-\frac{u+v}{2}, s\right)\right\} \\
& <N(y-z, 2 s),
\end{aligned}
$$

which is a contradiction. So at least one of the equalities holds in (2.1) and (2.2). Without lose of generality assume that equality holds in (2.1). Then

$N\left(y-\frac{u+v}{2}, s\right)=\max \{N(y-u, s), N(y-v, s)\}$

The strict convexity of $X$ implies that, $N(y-u, s)=N(y-v, s)$, and so, $u=v$. Therefore the proof is completed.

Theorem 2.2 Let $X$ and $Y$ be real fuzzy anti-normed spaces and let $Y$ be strictly convex. Suppose $f: X \rightarrow Y$ be a fuzzy isometry satisfies $f(x), f(y)$ and $f(z)$ are collinear when $x, y$ and $z$ are collinear. Then $f$ is affine.

Proof. Let $g(x):=f(x)-f(0)$. Then $g$ is fuzzy isometry and $g(0)=0$. It is easy to check that if $x, y$ and $z$ are collinear, then $g(x), g(y)$ and $g(z)$ are also collinear. So it suffices to show that $g$ is linear. We have

$N\left(g\left(\frac{y+z}{2}\right)-g(y), s\right)=N\left(\left(\frac{y+z}{2}\right)-y, s\right)=N(y-z, 2 s)$

and similarly

$N\left(g\left(\frac{y+z}{2}\right)-g(z), s\right)=N\left(\left(\frac{y+z}{2}\right)-z, s\right)=N(y-z, 2 s)$

for all $y, z \in X$ and $s>0$. By lemma (2.1) we have

$g\left(\frac{y+z}{2}\right)=\frac{1}{2} g(y)+\frac{1}{2} g(z)$.

Since $g(0)=0$, we can easily show that $g$ is additive. It follows that $g$ is $\mathcal{Q}$-linear. We have to show that $g$ is $\mathcal{R}$-linear.

Let $r \in \mathcal{R}^{+}$with $r \neq 1$ and $y \in X$. Since $0, y$ and $r y$ are collinear $g(0), g(y)$ and $g(r) y$ are also collinear. Since $g(0)=0$, there exists $r^{\prime} \in \mathcal{R}$ such that $g(r y)=r^{\prime} g(y)$. Now, we will proved that $r=r^{\prime}$. Since $y$ and $z$ are collinear, then $y \neq z$. Hence,

$$
\begin{aligned}
N(r(y-z), s) & =N(g(r y)-g(r z), s) \\
& =N\left(g\left(r^{\prime} y\right)-g\left(r^{\prime} z\right), s\right) \\
& =N\left(r^{\prime}(g(y)-g(z)), s\right) \\
& =N\left(r^{\prime}(y-z), s\right) .
\end{aligned}
$$

By the strict convexity we obtain $r(y-z)=r^{\prime}(y-z)$. Thus $g(r y)=r g(y)$ for all $y \in X$ and all $r \in \mathcal{R}$. Therefore $g$ is affine and the proof is complete.

\section{References}

[1] C. Felbin, "Finite dimensional fuzzy normed linear spaces", Fuzzy Sets and Systems, Vol.48, No.2, (1992), pp.239-248.

[2] T. Bag, S.K. Samanta, "A comparative study of fuzzy norms on a linear space", Fuzzy Sets and Systems, Vol.159, No.6, (2008), 670-684.

[3] J.A. Baker, "Isometries in normed spaces", Amer. Math. Monthly, Vol.78, No.6, (1971), 655-658.

[4] S.C. Cheng, J.N. Mordeson, "Fuzzy linear operator and fuzzy normed linear spaces", Bull. Calcutta Math. Soc. Vol.86, (1994), 429-436. 
[5] M. Eshaghi Gordji, N. Ghobadipour, "On the Mazur-Ulam theorem in fuzzy normed spaces", arXiv:0905.2166 (2009).

[6] Iqbal H. Jebril, T.K. Samanta, "Fuzzy anti-normed linear space", J. math. \& Tech., February (2010), 66-77.

[7] D. Kangb,.. H. Kohb, I. G. Choa, "On the Mazur-Ulam theorem in non-Archimedean fuzzy normed spaces", Appl. Math. Let. Vol.25, No.3, (2012), 301-304.

[8] S. Mazur, S. Ulam, "Sur les transformation isomtriques despaces vectoriels norms", C. R. Acad. Sci. Paris, Vol.194 (1932), 946-948.

[9] M. S. Moslehian and Gh. Sadeghi, "A Mazur-Ulam theorem in non-Archimedean normed spaces", Nonlinear Anal. Vol.69 (2008), 3405-3408.

[10] R. Saadati, S.M. Vaezpour, "Some results on fuzzy Banach spaces", J. Appl. Math. Computing, Vol.17, No. 1-2, (2005), 475-484.

[11] L.A. Zadeh, "Fuzzy sets", Inf. \& Cont., Vol.8 (1965), 338-353. 